\title{
A Novel Tomato Fusarium Wilt Tolerance Gene
}

\author{
Cahya Prihatna ${ }^{1,2 *}$, Martin J. Barbetti ${ }^{1,3}$ and Susan J. Barker ${ }^{1}$ \\ ${ }^{1}$ School of Agriculture and Environment, Faculty of Science, The University of Western Australia, Crawley, WA, Australia, \\ ${ }^{2}$ Research and Development for Biotechnology, PT Wilmar Benih Indonesia, Bekasi, Indonesia, ${ }^{3}$ The UWA Institute of \\ Agriculture, Faculty of Science, The University of Western Australia, Crawley, WA, Australia
}

The reduced mycorrhizal colonization $(r m c)$ tomato mutant is unable to form mycorrhiza and is more susceptible to Fusarium wilt compared with its wild-type isogenic line 76R. The $r m c$ mutant has a chromosomal deletion affecting five genes, one of which is similar to CYCLOPS. Loss of this gene is responsible for non-mycorrhizality in rmc but not enhanced Fusarium wilt susceptibility. Here, we describe assessment of a second gene in the rmc deletion, designated Solyc08g075770 that is expressed in roots. Sequence analyses show that Solyc08g075770 encodes a small transmembrane protein with putative phosphorylation and glycosylation sites. It is predicted to be localized in the plasma membrane and may function in transmembrane ion transport and/or as a cell surface receptor. Complementation and knock-out strategies were used to test its function. Some putative CRISPR/Cas-9 knock-out transgenic events exhibited Fusarium wilt susceptibility like rmc and some putative complementation lines were 76R-like, suggesting that the tomato Solyc08g075770 functions in Fusarium wilt tolerance. This is the first study to demonstrate that Solyc08g075770 is the contributor to the Tfw locus, conferring tolerance to Fusarium wilt in $76 \mathrm{R}$ which was lost in rmc.

Keywords: Fusarium wilt, tolerance, Solanum lycopersicum, Fusarium oxysporum f. sp. lycopersici, tomato transformation, mycorrhizal symbiosis

\section{INTRODUCTION}

Fusarium wilt disease in tomato, caused by Fusarium oxysporum f. sp. lycopersici (Sacc.) W.C. Snyder and H.N. Hans ( $\mathrm{Fol}$ ), is one of the most devastating diseases of tomato and has caused major losses in tomato production worldwide. The disease is characterized by wilting and browning of the leaves, yellowing, stunted growth, and eventual death of the plant. Crop yield is negligible in highly infected tomato plantings.

The interaction between $\mathrm{Fol}$ and tomato is race-cultivar specific. A classical gene-for-gene relationship has been proposed to underly the interaction between Fol races and their host cultivars, based on observations of dominant monogenic resistance triggered by challenge with known Fol races (Takken and Rep, 2010). Four resistance genes that confer resistance to known Fol races have been described in tomato: I; I-2; I-3; and I-7 (Bohn and Tucker, 1939; Stall and Walter, 1965; Simons et al., 1998; Catanzariti et al., 2015; Gonzalez-Cendales et al., 2016; Catanzariti et al., 2017). Three of these $\mathrm{R}$ genes have been incorporated into cultivated tomato from wild tomato species: the $I$ and $I-2$ genes from S. pimpinellifolium that confer resistance to Fol races 1 and 2, respectively, and the $I-3$ gene from S. pennellii, which confers resistance to Fol race 3 . In addition to the $I-3$ gene, resistance to Fol race 3 is also conferred by the $I-7$ gene (Gonzalez-Cendales et al., 2016). Moreover, I-7 also confers resistance to Fol races 1 and 2. It has been shown that Avr 1 in 
Fol race 1 suppresses the $I$-2- and $I$-3-mediated resistance (Houterman et al., 2008). Resistance to Fol race 1 indicates that $I$-7-mediated resistance, unlike $I$-2- or $I$-3-mediated resistance, is not suppressed by Avr1, giving further evidence that Avr1 does not suppress EDS1 (Enhanced Disease Susceptibility 1)dependent resistance conferred by the $I$ and $I-7$ genes (GonzalezCendales et al., 2016). I-3 is located on chromosome 7 and encodes an S-receptor-like kinase, whereas $I-7$ is on chromosome 8 and encodes a leucine-rich repeat receptor-like protein (LRRRLP) (Catanzariti et al., 2015; Gonzalez-Cendales et al., 2016). The $I$ gene has recently been identified and like $I-7$ it encodes a membrane-anchored LRR-RLP (Catanzariti et al., 2017).

In addition to major resistance conferred by resistance genes, some levels of tolerance or reduced susceptibility in tomato to $\mathrm{Fol}$ have been reported. Tolerance to Fol race 3 has been observed in heterozygous individuals of a S. lycopersicum $\times$ S. pennellii backcrossed population (Bournival et al., 1989). These individuals show moderate resistance to Fol race 3 compared to a population carrying the $I-3$ locus that is tightly linked to Got-2 marker on chromosome 7. This moderate disease resistance is linked to a fragment on chromosome 8 marked by Aps-2 and the locus linked to this marker is designated as $T f w$ (tolerance to Fusarium wilt).

The $r m c$ (reduced mycorrhizal colonization) tomato mutant is unable to form mycorrhizal symbiosis with most AM fungal species (Barker et al., 1998; Gao et al., 2001). In addition, rmc is also more susceptible to Fol race 3 compared with its wild-type isogenic line 76R (Barker et al., 2005). Therefore, the rmc mutation causes a contrasting phenotype of rootfungus interaction: colonization by AM fungi is impaired whereas Fol race 3 colonization is enhanced. The mutation is a deletion that disrupts five genes on chromosome 8, one of which shares sequence similarity to the CYCLOPS gene (Larkan et al., 2007, 2013). A separate study has confirmed that the CYCLOPS homolog is the gene that is responsible for the impaired AMF colonization in $r m c$, but does not influence Fusarium wilt tolerance (Prihatna et al., 2018). Thus, the enhanced susceptibility to Fol race 3 in rmc could be due to disruption of one of the other four genes. Interestingly, $R m c$ is located in the short arm of chromosome 8 that is linked to $A p s-2$ where the improved tolerance to Fusarium wilt (Tfw) is also located (Larkan et al., 2007). Thus it is likely that $T f w$ is one of the genes in the Rmc locus.

Of the other genes that are disrupted in $r m c$, Solyc08g075770 drew attention, being expressed in roots of $76 \mathrm{R}$ but not in rmc (Larkan et al., 2013). The predicted transcript sequence encodes a protein of unknown function. It shares sequence similarity with a predicted exopolysaccharide production negative regulator in Arabidopsis thaliana (Mayer et al., 1999). To dissect the genetic component leading to enhanced susceptibility to Fol race 3 in the $r m c$ phenotype, genetic complementation and CRISPR/Cas9-mediated gene knockout of Solyc08g075770 were undertaken. Furthermore, a rapid microscopy technique that allows functional analysis of tolerance in transgenic tomato to Fusarium wilt is described.

\section{MATERIALS AND METHODS}

\section{Tomato Genotypes}

The tomato $r m c$ line was generated by single seed descent from a fast neutron mutagenized population that was identified at the M2 generation (Barker et al., 1998). The parental, wild-type line '76R' (cv. Rio Grande) was obtained from Peto Seed Company, Saticoy, CA, United States. The $r m c$ tomato has a mutation spanning five genes on the short arm of chromosome 8 flanked by markers CT88 and EST248494 (Larkan et al., 2007, 2013).

\section{Fusarium oxysporum f. sp. lycopersici Races}

Fol races 1, 2, and 3 were used to test susceptibility of tomato $76 \mathrm{R}, \mathrm{rmc}$, and transformed lines. Fol race 1 (accession no. BRIP 17552a), Fol race 2 (accession no. BRIP 16848a), and Fol race 3 (accession no BRIP 15362a) were obtained from Queensland Plant Pathology Herbarium (Queensland Department of Agriculture, Fisheries, and Forestry). Fol BRIP 15362a originated from Dutton Creek, Bowen, QLD, Australia. Fol BRIP 16848a originated from Indooroopilly, QLD, Australia. Fol BRIP 17552a originated from Severnlea, QLD, Australia.

\section{Fusarium Wilt Assay}

$\mathrm{Fol}$ isolates were grown on potato dextrose agar for 10 days at room temperature $\left(\sim 25^{\circ} \mathrm{C}\right)$. The surface of the fungal cultures was then flooded with $5 \mathrm{ml}$ of sterile water containing $0.01 \%$ Tween $20^{\mathrm{TM}}$ and surface-scraped using a spatula to collect spores. Potato dextrose broth $(200 \mathrm{ml})$ in $500 \mathrm{ml}$ Erlenmeyer flasks was inoculated with $2 \mathrm{ml}$ of the spore suspension and incubated on a rotary shaker $(150 \mathrm{rpm})$ for 5 days at room temperature. After 5 days, spores were collected by filtering the culture through four layers of miracloth. The spore suspension was then centrifuged at $5,000 \times g$, supernatant was decanted and resuspended with sterile water. Spore concentration was counted using a hemocytometer (Improved Neubauer) and concentration adjusted to $1 \times 10^{7}$ spores $\mathrm{ml}^{-1}$ (Catanzariti et al., 2015).

Seeds of tomato $76 \mathrm{R}$ and $r m c$ were sown and germinated in pasteurized potting mix soil. Fourteen-day-old seedlings were uprooted and roots dipped into spore suspension for $3 \mathrm{~min}$, then returned to soil. Plants were watered twice daily and fertilized weekly using Thrive ${ }^{\mathrm{TM}}$ liquid complete fertilizer at the recommended rate. There were 10 replicate plants for each treatment, with each plant transplanted into a separate pot. For each tomato genotype, there were four treatments, i.e., Fol race 1, Fol race 2, Fol race 3, and uninoculated control. Plants were grown in a controlled environment room with $16 \mathrm{~h}$ photoperiod $\left(25^{\circ} \mathrm{C}\right.$ light and $22^{\circ}$ dark) at 600 Photosynthetic Photon Flux Density (PPFD) and humidity of $70 \%$ night (dark), $80 \%$ day (light). The experiments were repeated twice.

Disease was evaluated and scored 6 weeks after inoculation. Disease scoring was on a scale of 0-9 based on Fuchs et al. (1997) with modifications, as follows: 0 - healthy plant; 1 - mild chlorosis on lowest two leaves only, mild wilt; 2 - moderate chlorosis on lowest four leaves, mild wilt; 3 - moderate chlorosis on lowest four leaves and mild necrosis on lower two leaves; 
4 - moderate chlorosis on lowest four leaves and moderate necrosis on lower two leaves; 5 - moderate chlorosis on lowest six leaves and moderate necrosis on lower four leaves, severe on leaf two; 6 - moderate chlorosis on lowest six leaves and severe necrosis on four leaves, strong wilt; 7 - high chlorosis on lowest six leaves and severe necrosis on four leaves, strong wilt; 8 - plant almost dead, necrosis on all leaves and uppermost crown is chronically wilted; 9 - dead stump. The disease ranking is an ordered categorical (ordinal) variable that has non-Gaussian distribution, thus the statistical model applied was non-parametric. A generalized linear model was used to test the comparison of disease rankings between genotypes and/or Fol treatments. Probability was further adjusted using Tukey's HSD post hoc test. Statistical analysis was performed using the $\mathrm{R}$ statistics program (R Core Team, 2016). Fresh biomass of plants, crown and leaves were removed from soils and weighed. Statistical analyses were performed using $\mathrm{R}$ statistics program and significance of differences in biomass between genotypes and Fol races was assessed by two-way ANOVA $(P<0.05)$.

\section{T-DNA and Vector Constructions}

The Solyc08g075770 protein-coding sequence was cloned into pEarleyGate 100 (Earley et al., 2006) binary vector via Gateway cloning system to generate a binary vector pEarleyGateSolyc08g075770 (Supplementary Figure S1A). The binary vector clone was kindly donated by Daniel Ruzicka (Donald Danforth Plant Science Center, St. Louis, MO, United States). The Solyc08g075770 coding sequence is driven by a CaMV35S promoter with duplicated enhancer region $(2 \times 35 S)$. The vector harbors Basta ${ }^{\circledR}$ resistance gene (bar) for transgenic plant selection and kanamycin resistance gene cassette (nptII and KanR) for bacterial selection.

For genome editing using CRISPR/Cas9, single-guide RNAs (sgRNAs, or protospacers) were designed from genomic sequence of Solyc08g075770 gene using Benchling, an online molecular biology suite ${ }^{1}$. Construction and cloning of sgRNA into a binary vector was conducted based on methods described by Schiml et al. (2014). The sgRNA was cloned into an entry vector pEn-C1.1 using BbsI restriction site so that it was inserted between U6-26 promoter and sgRNA scaffold. The U6-26 promoter:sgRNA:sgRNA scaffold cassette was then cloned using Gateway Cloning system into a destination vector pDe-CAS9 to generate a binary vector pRMC-CAS9 (Supplementary Figures S1B,C). pEn-C1.1 and pDe-CAS9 were kindly provided by Holger Puchta (Botanical Institute II, Karlsruhe Institute of Technology, Germany) (Schiml et al., 2014).

\section{Bioinformatics of Solyc08g075770 Gene}

The protein coding sequence (cDNA) of Solyc08g075770 was translated into protein sequence with the standard genetic code using Translate tool ${ }^{2}$. The resulting protein sequence was used for sequence analyses. The amino acid sequence of Solyc08g075770 was queried in BLAST protein (BLASTP) against non-redundant (nr) protein sequence database containing entries

${ }^{1}$ https://benchling.com/

${ }^{2}$ http://web.expasy.org/translate/ from some members of Solanaceae, Brassicaceae, Fabaceae, Poaceae, Orchidaceae, gymnosperm lycophyte and moss. Protein sequences retrieved from BLASTP hits were downloaded and aligned using Clustal Omega (Sievers et al., 2011) and visualized using JalView (Waterhouse et al., 2009). A phylogenetic tree was built from the aligned sequences and inferred by using Maximum Likelihood method with 500 bootstraps (Jones et al., 1992). These evolutionary analyses were conducted in MEGA7 (Kumar et al., 2016).

Protein sequences from some members of the Solanaceae family were then aligned using Clustal Omega. Structural and functional components were assessed using available bioinformatics tools. Transmembrane helices in Solyc08g075770 were predicted using Hidden Markov Model via TMHMM Server v. 2.03 . Homology modeling was attempted using SWISS-MODEL ${ }^{4}$ (Biasini et al., 2014). Template was searched using BLAST against the SWISS-MODEL template library and templates with highest quality selected for model building using ProMod-II (Guex et al., 2009). Overall structure prediction and gene ontology were performed using PSIPRED Protein Sequence Analysis Workbench ${ }^{5}$ and AmiGO $2^{6}$. Functional motifs and post-translational modifications were predicted using PROSITE $^{7}$.

\section{Tomato Transformation Procedure}

Agrobacterium tumefaciens strain AGL1 (Lazo et al., 1991) was used to transform $r m c$ tomato for genetic complementation and 76R tomato for CRISPR/Cas9 sequence modification. For transformation, cotyledons of 10-day-old tomato seedlings were cut at the distal and proximal ends and the middle parts were used as transformation explants. The explants were pre-cultured on co-cultivation medium (CCM) for $24 \mathrm{~h}$ in the dark at $25^{\circ} \mathrm{C}$ and then inoculated with AGL1 cell suspension (OD600 $=0.2$ ). The inoculated explants were returned to CCM and incubated for $48 \mathrm{~h}$ in the dark at $25^{\circ} \mathrm{C}$. After $48 \mathrm{~h}$, explants were washed with timentin solution ( $300 \mathrm{mg} \mathrm{l}^{-1}$ ) several times to remove bacterial cells and transferred onto pre-selection, shoot induction medium (SIM1) without plant selection and incubated for 7 days at $25^{\circ} \mathrm{C}$ in white, cool light fluorescence [150 PPFD $\left.\left(\mu \mathrm{mol} \mathrm{m}{ }^{-2} \mathrm{~s}^{-1}\right)\right]$. After a week, explants were transferred to second shoot induction medium (SIM2) which comprised of SIM1 containing $0.5 \mathrm{mg} \mathrm{l}^{-1}$ phosphinothricin, to select resistant, transformed cells. Calli and shoots were cultured on this medium for 4 weeks and refreshed onto new medium every week. Healthy calli were isolated from necrotic tissues to prevent cross-poisoning from dying to healthy tissues. At this stage, developed shoots were transferred to shoot elongation medium (SEM) until shoots were about $2.5 \mathrm{~cm}$ in length prior to transferring to a rooting medium (RM). Surviving explants and calli were transferred back to SIM1 to recover and regenerate more shoot primordia. Composition of media used for tissue cultures in this study is provided in Supplementary Table S1.

\footnotetext{
${ }^{3}$ http://www.cbs.dtu.dk/services/TMHMM/

${ }^{4}$ http://swissmodel.expasy.org/

${ }^{5}$ http://bioinf.cs.ucl.ac.uk/psipred/

${ }^{6}$ http://amigo.geneontology.org/amigo

${ }^{7}$ http://prosite.expasy.org/
} 


\section{Evaluation of Putative Transgenic Events}

Genomic DNA of regenerated plantlets in tissue culture was analyzed by PCR. Genomic DNA was extracted using a cetyltrimethylammonium bromide (CTAB) method adapted from Murray and Thompson (1980). To detect genetic complementation of $r m c$ with Solyc08g075770, primers AKCDS4$\mathrm{F}$ and AKCDS4-R were used to amplify $174 \mathrm{bp}$ fragment of Solyc08g075770 coding sequence in complemented $r m c$ and $888 \mathrm{bp}$ fragment of non-mutant $(R m c, 76 \mathrm{R})$ genomic DNA. Thus, these primers can differentiate genuine transgenic $r m c$ events from $76 \mathrm{R}$ contamination. Act2F-Act2R primers were used to amplify the actin gene as a positive PCR control. All PCR was performed using MyTaq ${ }^{\mathrm{TM}}$ Red Mix (BIOLINE). PCR conditions were as follows: initial denaturation at $95^{\circ} \mathrm{C}$ for $3 \mathrm{~min}$ followed by 35 cycles of denaturation at $95^{\circ} \mathrm{C}$ for $30 \mathrm{~s}$, primer annealing at $56^{\circ} \mathrm{C}$ for $30 \mathrm{~s}$, and polymerisation at $72^{\circ} \mathrm{C}$ for $50 \mathrm{~s}$. The PCR was finalized by polymerisation at $72^{\circ} \mathrm{C}$ for $5 \mathrm{~min}$.

To detect CRISPR/Cas9 transgenic 76R events, Cas9 gene was amplified using primers Cas9F1 and Cas9R1. Concomitant with PCR of Cas9, a genomic fragment covering the protospacer region was amplified using primers CRISPR_F1 and CRISPR_R1 and the amplicons were then Sanger-sequenced using the same primers. The Solyc08g075770 genomic region of $76 \mathrm{R}$ was also amplified and sequenced. The sequenced DNA was then analyzed for any mutations.

To ensure there was no genotype contamination, i.e., 76R contamination in the complemented $r m c$ and $r m c$ in the CRISPR/Cas9 genome editing of 76R, an additional PCR was performed to amplify CYCLOPS in all plants used for examination. Hence, CYCLOPS is amplified from 76R but not from rmc. Primers used to amplify CYCLOPS were RmcCDS1S and RmcCDS1A. The sequences of the primers used in this study are provided in Supplementary Table S2.

\section{Microscopy of Root Colonization}

Tissue-culture-grown 76R, $r m c$, and putative transformed plants were uprooted from rooting agar media and the roots washed several times using sterile water to remove agar from the roots. Plants were then put into glass jars containing Fol race 3 spore suspension at a concentration of $10^{7}$ spores $\mathrm{ml}^{-1}$ in water. Plants were leaned against the walls of the jars with the roots immersed in spore suspension. One plant from each genotype was put in one jar and there were three replicates for each plant genotype. The experiments were fully repeated twice. The jars were incubated in a growth chamber for 7 days at $25^{\circ} \mathrm{C}$ in white, cool light fluorescence [150 PPFD $\left(\mu \mathrm{mol} \mathrm{m}{ }^{-2} \mathrm{~s}^{-1}\right)$ ].

Roots were examined for colonization every $12 \mathrm{~h}$ using a light compound microscope (Leica DM4000 B LED, Leica Microsystems CMS GmbH). For each observation, at least six roots were cut at approximately $1-\mathrm{cm}$-length and then stained by ink-vinegar as described in Vierheilig et al. (1998) with some modifications. Root materials were harvested and cleared using $10 \%(\mathrm{w} / \mathrm{v}) \mathrm{KOH}$ at $55^{\circ} \mathrm{C}$ overnight. Cleared roots were rinsed several times with water and then immersed in $0.1 \mathrm{~N}$ $\mathrm{HCl}$ for approximately $30 \mathrm{~s}$ or until the roots appeared slightly lighter in color. Acidified roots were then stained using an ink-vinegar solution [5\% (v/v) Parker Quink ink in white vinegar] for $1 \mathrm{~h}$ at $55^{\circ} \mathrm{C}$ followed by $1 \mathrm{~h}$ at room temperature. Roots were then destained in a $5 \%(\mathrm{v} / \mathrm{v})$ vinegar solution for $30 \mathrm{~min}$ and washed several times with water and stored in lactoglycerol (lactic acid: glycerol: $\mathrm{dH}_{2} \mathrm{O}=1: 1: 1, \mathrm{v} / \mathrm{v} / \mathrm{v}$ ). Roots were observed using phase-contrast microscopy with $40 \times, 100 \times$, $200 \times$, and $400 \times$ magnifications. Roots were scanned starting from root tips and then moving away toward distal parts of roots sequentially to span at least 10 overlapping regions of each root.

To quantify the levels of Fol colonization on the roots, total area of mycelia was measured and calculated against the total area of each image and expressed as percentage. The analysis was performed using ImageJ (Schindelin et al., 2012). The redgreen-blue (true color) channels of images were first split into 8 -bit images to obtain grayscale images. To highlight the area of interest, i.e., hyphae/mycelia, the threshold of the images was adjusted and then measurements were performed on the images with parameters delimited by the threshold values.

\section{RESULTS}

\section{rmc Tomato Mutant Is More Susceptible to Fol Race 3 Compared to $76 R$}

Fusarium wilt bioassay in this study confirmed the results from a previous study that shows $r m c$ is more susceptible to Fol race 3 compared with $76 \mathrm{R}$ but that both tomato genotypes were resistant to Fol race 1 (Barker et al., 2005). Early symptoms of wilt appeared at 2 weeks post inoculation (wpi) and were yellowing of older leaves and stunted growth. At three wpi, growth of $r m c$ and 76R plants inoculated with Fol race 3 was severely stunted compared with non-inoculated controls and plants inoculated with Fol race 1 (Figures 1A-F). Further, growth of $r m c$ inoculated with Fol race 3 was also significantly more stunted compared with 76R plants (Figures 1E,F).

At six wpi, some $r m c$ plants inoculated with $\mathrm{Fol}$ race 3 had died but no dead 76R plants were observed. Although most of the plants were severely wilted and stunted, $r m c$ plants had more severe wilting and necrotic leaves compared with $76 \mathrm{R}$ plants (Figure 2A). The fresh biomass of $r m c$ plants was also lower compared to $76 \mathrm{R}$ although the difference was not significant $(P>0.05)$ (Figure 2B).

\section{Solyc08g075770 Encodes a Small Transmembrane Protein With Predicted Phosphorylation Sites}

The predicted protein coding sequence of Solyc08g075770 gene has 906 base pairs and a search of the tomato genome ${ }^{8}$ identified only one copy of this gene in the tomato genome. It encodes a protein with 301 amino acid sequence with unknown function. The coding sequence was derived from the predicted five exons in the $9.8 \mathrm{~kb}$ of gene sequence including the $5^{\prime}$ and $3^{\prime}$ untranslated regions (UTRs) as shown in Supplementary Figure S1C. The gene

\footnotetext{
${ }^{8}$ https://solgenomics.net/
} 


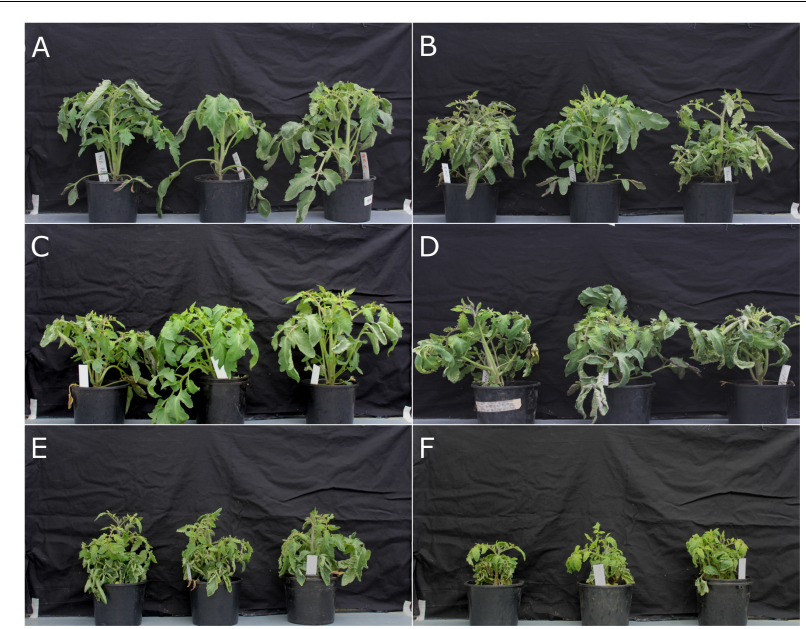

FIGURE 1 | Fusarium wilt disease assay on rmc and 76R tomatoes confirming $r m c$ as more susceptible to Fusarium oxysporum f. sp. Iycopersic (Fol) race 3 than 76R. (A) Tomato $76 \mathrm{R}$ and (B) $r m c$ were inoculated with Fol race 1 ; (C,D) $76 \mathrm{R}$ and $r m c$ were inoculated with $\mathrm{Fol}$ race 2; (E,F) $76 \mathrm{R}$ and rmc were inoculated with Fol race 3 . Plants were inoculated using a root dip method and photographed 3 weeks after inoculation.

is located adjacent to a gene that shares sequence similarity to CYCLOPS and a predicted ubiquitin-fold modifier 1 gene.

BLAST search on amino acid sequence of Solyc08g075770 revealed broad sequence similarity across a wide range of plant species including plants of primitive lineages, i.e., the moss Physcomitrella patens and the early diverging vascular plant Selaginella moellendorffii. The evolutionary tree of Solyc08g075770 in the extant modern plants showed that it has evolved in a distinct pattern from the sequence in extant early land plants, then it further diverged with clear distinction between monocots and dicots (Figure 3A).

Solyc08g075770 is predicted to be a membrane-bound protein that contains three transmembrane domains with cytoplasmic (intracellular) glycosylation and kinase phosphorylation sites (Figure 3B). There was no nuclear localization signal predicted from the protein sequence. The amino acid sequences are highly conserved amongst Solanaceae plants (Figure 3C). Alignment of amino acid sequences in plants that represented dicots (Solanaceae, Brassicaceae, and Fabaceae), monocots (Poaceae and Orchidaceae), gymnosperm, moss, and lycophyte showed less sequence conservation (Supplementary Figure S2). However, some predicted phosphorylation and glycosylation sites were present in all these sequences and this cytoplasmic region is relatively more conserved across the plant species that were assessed. These include phosphorylation motifs V-D-[LIV]$\mathrm{R}[\mathrm{SV}]-[\mathrm{SA}]-\mathrm{K}-[\mathrm{VTI}]-\mathrm{C}$ and [ERGK]-[TSV]-Y and glycosylation site C-R-P-[NDSVE]-F-[GSAD]. On the other hand, the leading amino acid sequence (N-termini) in the predicted extracellular region was the least conserved, even among the Solanaceae (Figure 3C and Supplementary Figure S2). The tomato Solyc08g075770 shared moderate sequence similarity with an exopolysaccharide production negative regulator AT4G19140
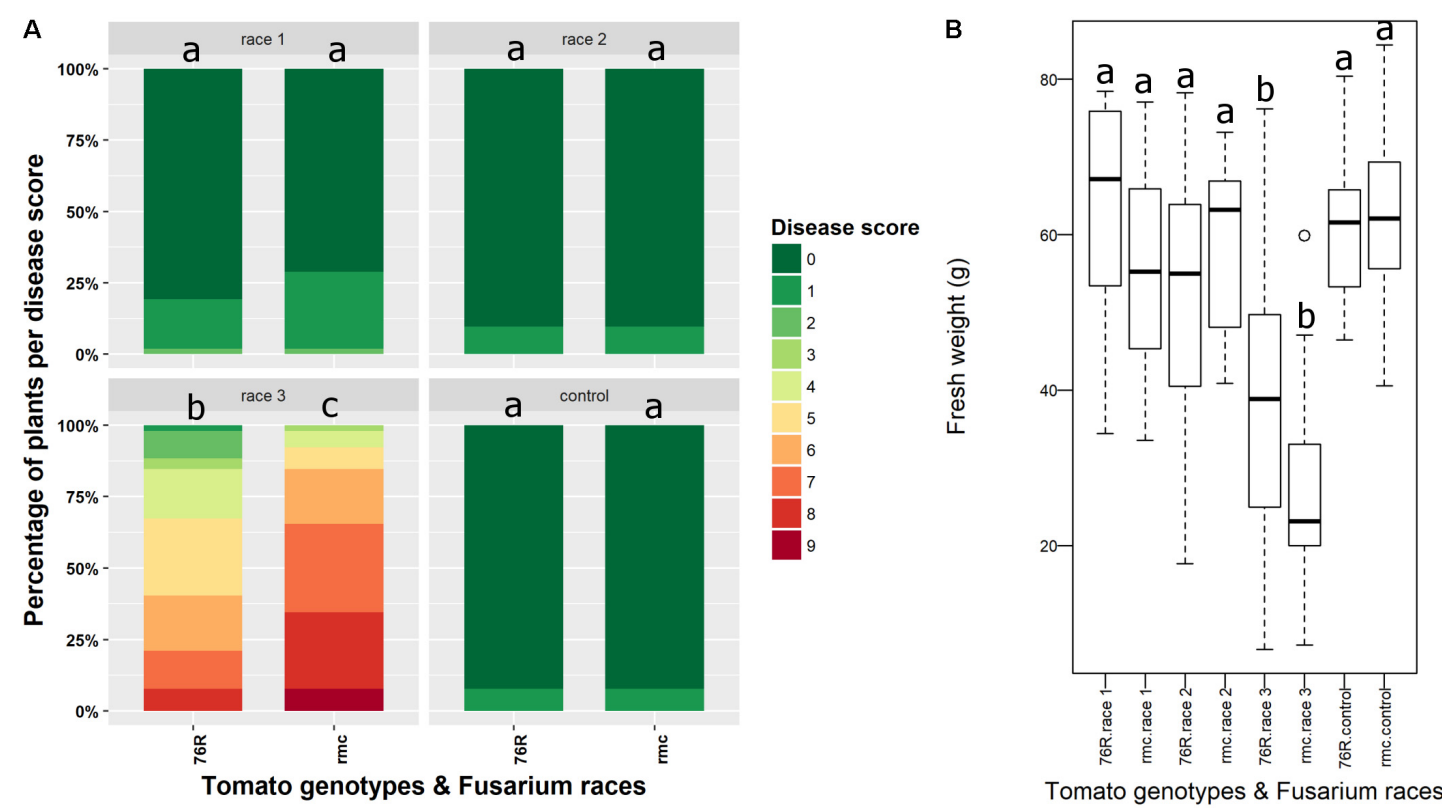

FIGURE 2 | Fusarium wilt disease assessment in rmc and 76R 6 weeks after inoculation with Fusarium oxysporum f. sp. Iycopersici (Fol) race 1, race 2, and race 3. (A) Disease scoring of Fusarium wilt according to Fuchs et al. (1997) at 6 weeks after inoculation. The area for each color code indicates the percentage of plants having the corresponding disease scores. At least 10 plants of either rmc or 76R plant type in an individual pot were used in this assay. Control is non-inoculated plants treated only with water. Stacked bars followed by the same letter are not significantly different at the $P<0.05$ level. (B) Box plot analysis showing that fresh weight of $r m c$ plants inoculated with Fol race 3 is lower than $76 \mathrm{R}$ inoculated with Fol race 3 although the difference is not significant $(P>0.05)$. Box plots indicated by the same letters are not significantly different at the $P<0.05$ level. 
A

A Solanaceae

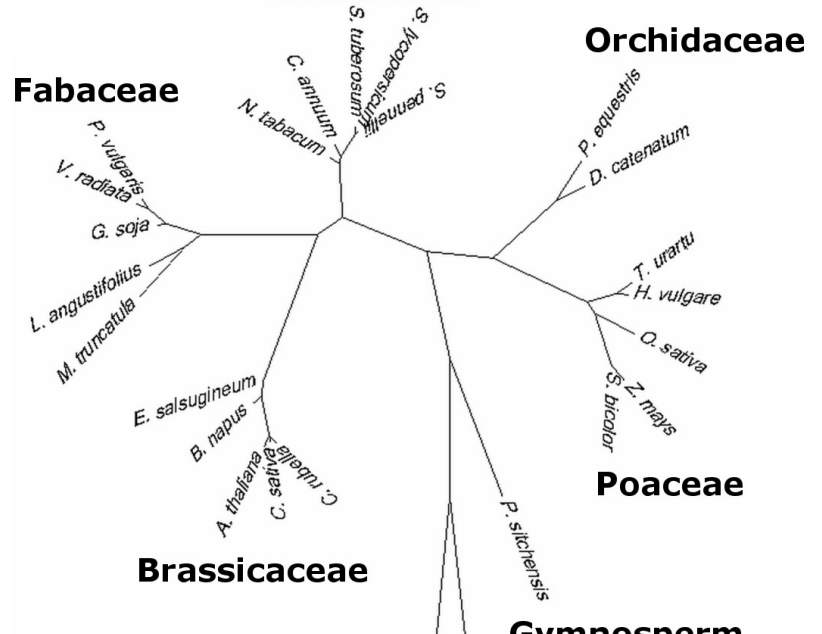

B

Gymnosperm
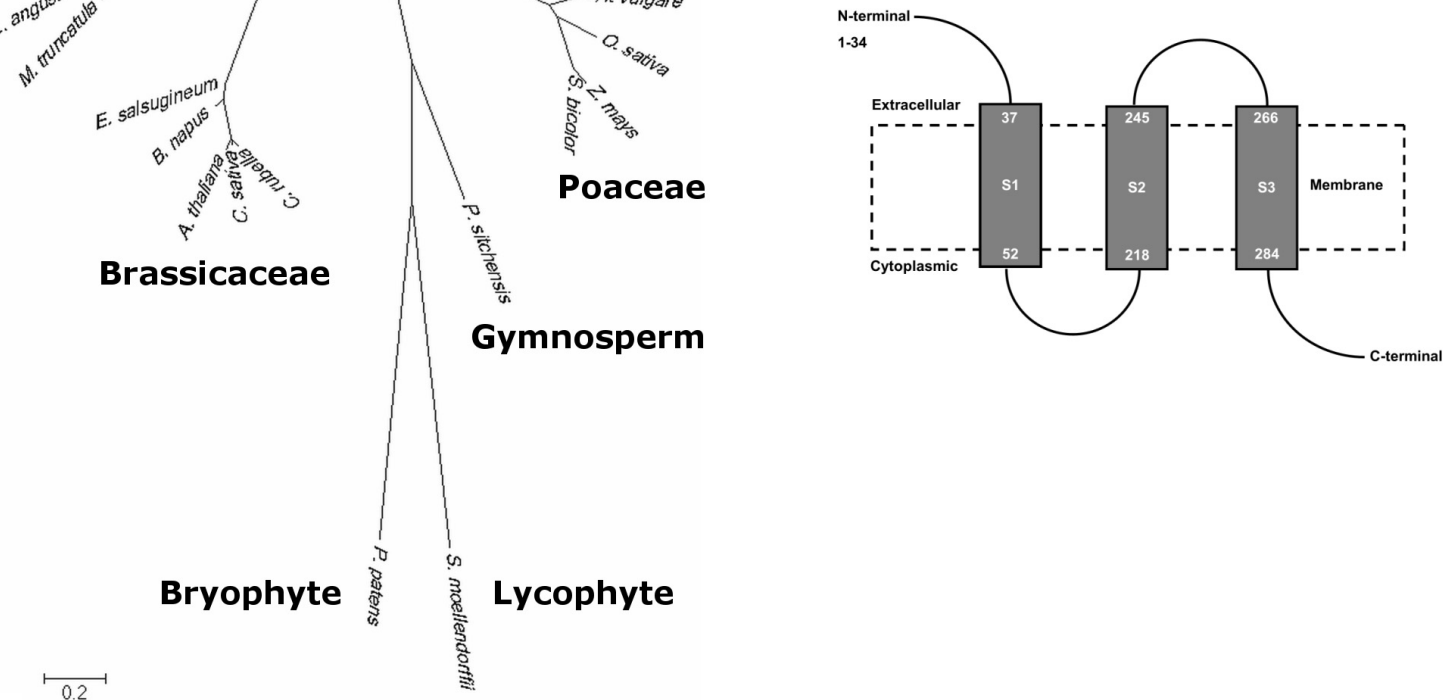

C
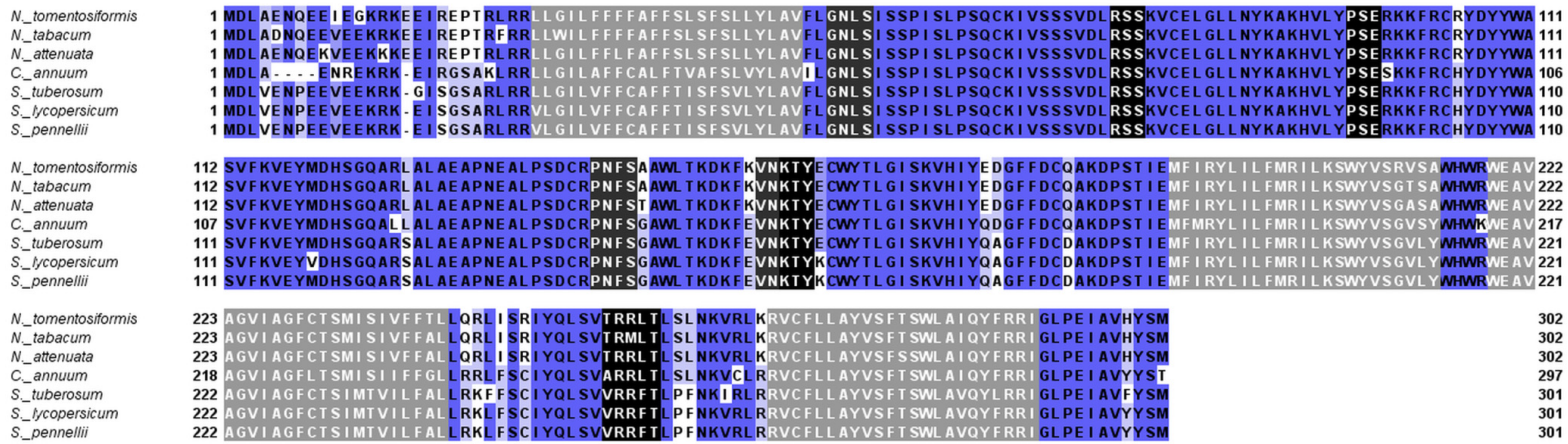
107 SVFKVEYMDHSGQALL AL AE APNEALPSDCRPNF SAWL. TKDKFEVNKT
111 SVFKVEYMDHSGQARSALAEAPNE ALPSDCRPNF SGAWL.TKDKFEVNKT

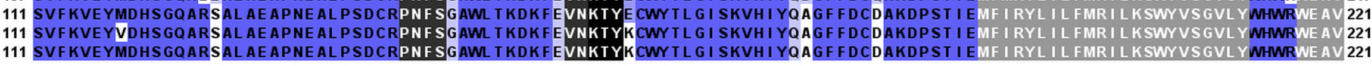

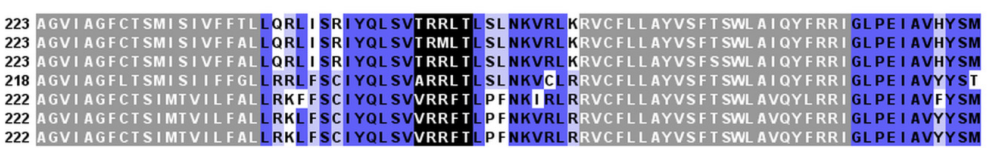

302
302
302
297
301
301
301

FIGURE 3 | Amino acid sequence analyses of Solyc08g075770. (A) Molecular phylogenetic analysis by Maximum Likelihood method of Solyc08g075770 and similar sequences in different plant species with 500 bootstrap replications. The tree is drawn to scale, with branch lengths measured in the number of substitutions per site. (B) Solyc08g075770 is predicted to be a protein having three transmembrane domains. Gray boxes are the transmembrane domains, and dashed box is an illustrated cell membrane. (C) Multiple alignment of amino acid sequences of Solyc08g075770 and similar sequences in several plants of the Solanaceae family. Sequences that are shaded gray are the predicted transmembrane domains; sequences that are shaded black are phosphorylation sites whereas sequences with dark gray shading are glycosylation sites (Nicotiana tomentosiformis XM_009622423, N. tabacum XM_016650100, N. attenuata XM_019371074, Capsicum annuum XM_016717203, Solanum tuberosum XM_006364398, S. pennellii XM_015227859). Sequences that are shaded blue are sequences that have significant similarity. The intensity of blue color indicates the degree of conservation or sequence identity: the darker the blue the more conserved the sequences.

in A. thaliana chromosome 4 (data not shown) (Mayer et al., 1999). Based on homology modeling, Solyc08g075770 is predicted to have a role in ion transmembrane channel/transport or in a cell surface receptor signaling pathway. Specifically, gene ontology revealed that Solyc08g075770 shared similarity with voltage-gated ion channel and adenylate cyclase-inhibiting G-protein coupled glutamate receptor sequences (Supplementary Table S3).
Search on gene transcripts was conducted to retrieve sequences similar to Solyc08g075770 that are expressed in roots of tomato induced by $\mathrm{Fol}$ infection. BLAST search on expressed sequence tag (EST) database in GenBank retrieved four ESTs that were related to Solyc08g075770 mRNA. These ESTs were FS202019.1, FS189001.1, BI421171.1, and AW441556.1. Of particular interest were ESTs FS202019 and FS189001 that were expressed in roots of tomato Micro-Tom treated with Fol race 2 
(Aoki et al., 2010). These ESTs (FS202019, cDNA library clone LEFL3149B10; FS189001, cDNA library clone LEFL3062C18) sequences matched the Solyc08g075770 gene. The EST FS202019 contains the full-length sequence of Solyc08g075770 mRNA (cDNA library clone LEFL3149B10). The other ESTs, BI421171 and AW441556, were retrieved from EST database of GenBank and these sequences were found in callus and ripe fruit of tomato, respectively (Alcala et al., unpublished).

\section{Assessment of the Role of Solyc08g075770 in Tolerance of Tomato to Fusarium Wilt by a Rapid Microscopy Assay}

To determine the role of Solyc08g075770 gene in tolerance to Fusarium wilt, genetic complementation in $r m c$ and gene knockout in 76R through CRISPR/Cas9 were attempted. There were seven putative complemented $r m c$ and 12 putative CRISPR/Cas9 knocked-out mutant events obtained from Agrobacteriummediated transformation. Of these 12 putative CRISPR/Cas9 knocked-out mutants, four did not produce normal shoots and failed to produce enough root materials for testing. Thus, they were discarded, and the remaining eight events were used in this experiment. To facilitate rapid screening of transgenic materials, a microscopy assay was developed that examined the early stages of susceptible Fusarium wilt colonization $(\mathrm{rmc})$ compared to Fusarium wilt tolerance (76R). Microscopy scanning on at least 20 roots for each $r m c$ and 76R lines revealed that roots of $r m c$ were consistently colonized more heavily compared with 76R (Figures 4A,B, 5 and Supplementary Table S4). In both $76 \mathrm{R}$ and $r m c$, colonization on roots was observed as soon as $12 \mathrm{~h}$ after inoculation. At this stage, hyphae were frequently found in the root hairs and less frequently on the root surface. After $24 \mathrm{~h}$, hyphae had colonized root surfaces more intensely and root apex colonization had also commenced. Fol race 3 preferentially colonized the elongation and maturation zones of the roots with occasional colonization at the apical meristem. The intensity of colonization gradually decreased toward distal (older) parts of the roots. The difference in the root colonization between $76 \mathrm{R}$ and $r m c$ was not so much in the frequency of the infection sites, but instead there was a marked difference in the abundance of hyphal growth, with more mass of $\mathrm{Fol}$ race 3 mycelia on $\mathrm{rmc}$ roots compared with 76R (Figures 4, 5 and Supplementary Table S4).

The extent to which putative complemented $r m c$ transgenic events were colonized by Fol race 3 was comparable, but not identical, to 76R. Two out of seven putative complemented $r m c$ showed the expected phenotype of restored levels of tolerance as in 76R if complementation occurred (events 26AK02 and 26AK11). The other five putative events resembled $r m c$, which is indicative of non-complemented $r m c$. The 26AK11 event resembled lightly colonized $r m c$ whereas the 26AK02 resembled slightly heavier colonized 76R (Figures 4C, 5 and Supplementary Figure S3). Two out of eight of the putative knock-out mutants (events CRISPR01 and CRISPR02) exhibited $r m c$-like phenotype with heavy Fol colonization (Figures 4D, 5 and Supplementary Figure S3) indicative of genuine knockout mutants, whilst other putative knock-out mutant events resembled 76R.

\section{Molecular Detection of Transformed Tomato Plants}

In addition to the rapid microscopy assay, screening of putative transformed plants was conducted by detecting transgene using PCR. PCR amplification of Solyc08g075770 from several putative complemented $r m c$ plants showed either specific but faint signal or non-specific but strong bands of amplicon DNA (Supplementary Figure S4). For the putative complemented $r m c$ that showed 76R-like root colonization phenotype (26AK02 and 26AK11), these events also showed relatively stronger bands of amplicon DNA (Supplementary Figure S4A). For CRISPR/Cas9 event analysis, Cas9 fragment was amplified from several plants (Supplementary Figure S4). Similarly, the putative knock-out mutants CRISPR01 and CRISPR02, which exhibited $r m c$-like phenotype, showed relatively stronger bands of Cas9 amplicon compared with the other putative mutants (Supplementary Figure S4B). However, the sequence of some putative CRISPR/Cas9 knock-out mutants showed there was no mutation in and around the sgRNA region, the same as in the wild-type 76R (Supplementary Figure S5). These potentially transgenic plants, that had the hall mark control PCR band amplification of their intended parent, were examined for root colonization by Fol race 3 .

\section{DISCUSSION}

This is the first study to assess the role of the Solyc08g075770 gene in Fusarium wilt tolerance of tomato. We had hypothesized that the gene is likely involved in root interactions with microbes because of its localized expression in roots and proximal location to a gene that shares similarity to CYCLOPS which in other plant species is involved in mycorrhizal symbiosis. We predicted that the Solyc08g075770 gene encodes a small transmembrane protein containing glycosylation and phosphorylation motifs. Functional analyses through genetic complementation and CRISPR/Cas9 provided supportive evidence of the role of Solyc08g075770 in Fusarium wilt tolerance. Observation on root colonization by Fol race 3 using a rapid microscopy assay highlighted presence of expected phenotypes in complemented $r m c$ and knockedout 76R, further indicating that Solyc08g075770 functions in tolerance to Fusarium wilt. Together, the results presented in the current study demonstrate that Solyc08g075770 is the contributor to the Tfw locus identified by Bournival et al. (1989), conferring tolerance to Fol race 3 in 76R which was lost in $r m c$.

The presence of amino acid coding sequences similar to Solyc08g075770 in moss and lycophyte genomes suggested that the Solyc08g075770 has been conserved in evolution since early colonization of land with plants. Unlike the tomato CYCLOPS, the Solyc08g075770 gene is also present in the nonmycorrhizal plant A. thaliana. In A. thaliana, the gene is predicted to encode an exopolysaccharide production negative regulator (Mayer et al., 1999). Alignment of Solyc08g075770 with 


\section{A}
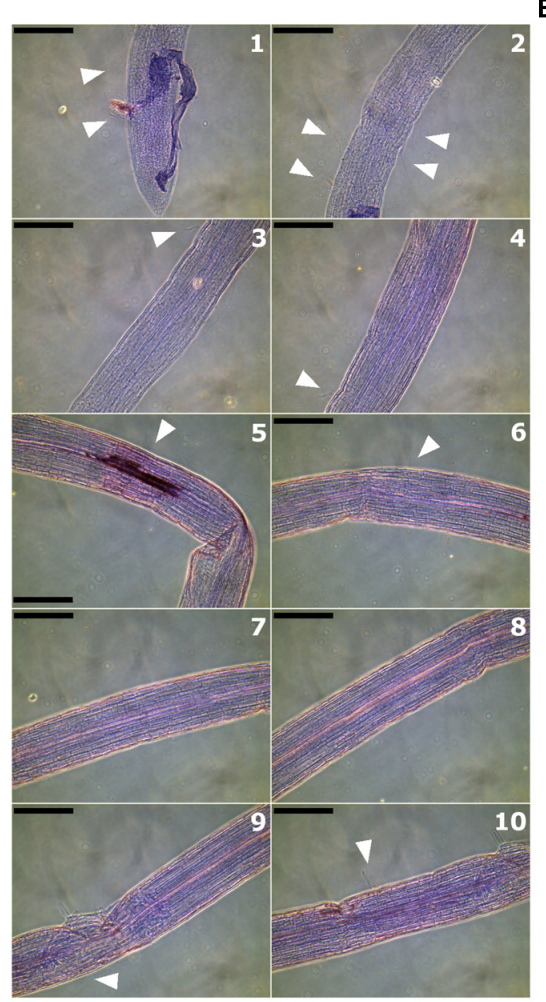

.
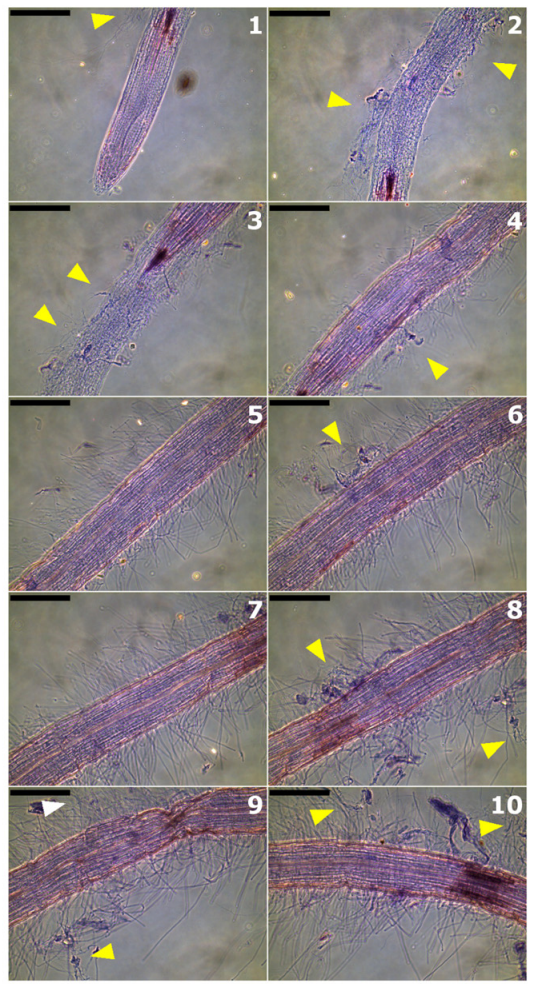

C

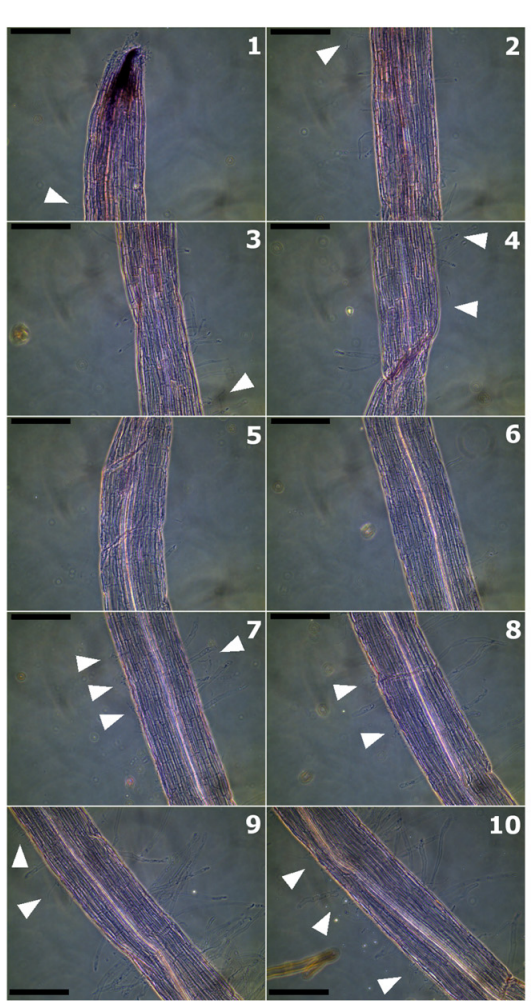

D

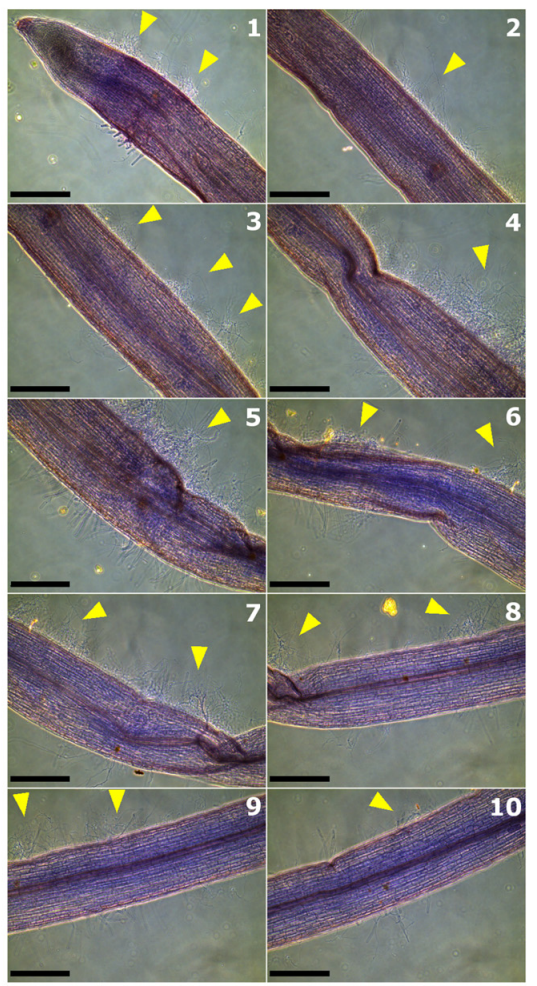

FIGURE 4 | Root colonization by Fusarium oxysporum f. sp. lycopersici (Fol) race 3 observed by phase-contrast microscopy at 24 h after inoculation. Roots were scanned at 10 overlapping zones starting from root tips and moving toward the crown. Numbers in each panel denote the sequential root zones. Light or mild colonization is shown by white arrows whereas heavy colonization is shown by yellow arrows. (A) Colonization of roots of 76R; (B) root colonization in rmc; (C) root colonization in 26AK02, a putative complemented rmc; and (D) root colonization in CRISPR01, a putative knock-out $76 \mathrm{R}$. Scale bars = $200 \mu \mathrm{m}$. 


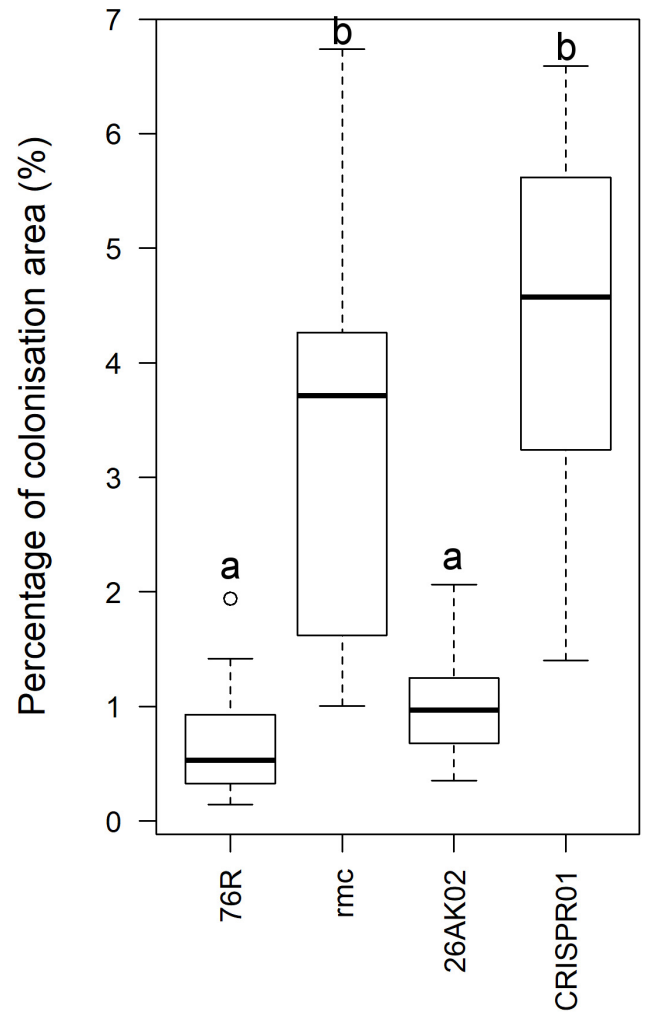

FIGURE 5 | Quantitative analysis of intensity of root colonization by Fusarium oxysporum f. sp. lycopersici (Fol) race 3 observed by phase-contrast microscopy at $24 \mathrm{~h}$ after inoculation. Quantitative analysis was performed using ImageJ (Schindelin et al., 2012). Intensity of colonization was measured as area of mycelia against the total area of image. Image quantification was based on gray levels of 8-bit images. Box plot indicated by the same letters are not significantly different $(P<0.001)$.

several similar sequences in Solanaceae plant genomes allowed the identification of putative transmembrane domains in the Solyc08g075770. Since there is no nuclear localization signal detected, Solyc08g075770 could be an integral component of plasma and/or organelle membranes.

The predicted phosphorylation sites in the cytoplasmic region of Solyc08g075770 suggest that it is a substrate of kinase involved in the signaling pathway. Interestingly, the polymorphic region is located at the leading sequence ( $\mathrm{N}$-terminal) which is predicted to be located extracellularly. This suggests that the polymorphic extracellular region could be involved in recognition of biotic or abiotic cues or patterns. However, the lack of putative conserved domains in Solyc08g075770 has made it difficult to predict its function or role in plant-microbe interactions. In particular, Solyc08g075770 lacks the leucine-rich repeat (LRR) and kinase domains (KD) in the LRR-receptor-like kinase (RLK) which are the most widespread protein domains that function in microbial perception and signal transduction (Wei et al., 2015; Liu et al., 2017). Nevertheless, structural modeling predicted that Solyc08g075770 could be an ion channel protein. The role of ion channels in plant immunity has been reported in cyclic nucleotide-gated ion channels (CNGCs)
(Moeder et al., 2011). These channel proteins are involved in pathogen-inducible $\mathrm{Ca}^{2+}$ influx, which in turn induces reactive oxygen species and mitogen-activated protein kinase (MAPK) cascades in plant defense responses. In higher plants, there are no canonical $\mathrm{Ca}^{2+}$ voltage-gated ion channels, but glutamate receptors, together with CNGCs, have been reported (Dietrich et al., 2010). Solyc08g075770 is also predicted to be a G-protein coupled glutamate receptor, suggesting a potential involvement of Solyc08g075770 in $\mathrm{Ca}^{2+}$ influx.

Functional analysis of Solyc08g075770 was determined by microscopic observation on root colonization by Fol race 3 . The consistent difference in root colonization between $r m c$ and 76R indicates that this method provides a rapid and reliable assessment to differentiate the phenotypes between $r m c$ and $76 \mathrm{R}$ in relation to Fol race 3 tolerance or susceptibility. In the traditional disease assay, the more heavily colonized $r m c$ roots observed by microscopy correlated with enhanced susceptibility in $r m c$ compared with 76R. Results from microscopic observation showed that some putative transformed plants (26AK02, 26AK11, CRISPR01, and CRISPR02) exhibited the expected phenotypes if the plant transformation was successful and thus evidence that Solyc08g075770 has a role in Fusarium wilt tolerance.

Two putative complemented $r m c$ plants (26AK02 and 26AK11) showed light root colonization similar to $76 \mathrm{R}$ and two putative knock-out 76R plants (CRISPR01 and CRISPR02) showed heavy colonization similar to $r m c$. However, scanning of roots across at least 10 overlapping regions, starting from root tip then moving away farther to the distal parts of the roots, minimized any chance of random colonization patterns. It was shown that colonization occurred mainly at the elongation and maturation regions of the roots, whereas the root apex, although it was often colonized, was less heavily colonized. Overall, this pattern of colonization is consistent with the findings of Olivain and Alabouvette (1999) who showed that at $24 \mathrm{~h}$ after inoculation roots were already colonized mainly at the elongation and mature zone of roots and that the intensity of colonization decreased progressively toward root tip and distal zone. Although internal root colonization was not observed in the current study, the correlation between heavier colonization and increased susceptibility in $r m c$ compared with $76 \mathrm{R}$ strongly suggests that the extent of root colonization by $\mathrm{Fol}$ race 3 can predict the levels of tolerance to this pathogen.

The finding of two ESTs (FS202019 and FS189001), representing Solyc08g075770 in roots of tomato MicroTom that were induced by infection of $\mathrm{Fol}$ race 2, signifies likelihood that this gene is involved in tomato-Fol interaction. Furthermore, $r m c$ is located on a small region in the short arm of chromosome 8 that is linked to Aps-2 (Larkan et al., 2007), as is a locus that confers improved tolerance to Fusarium wilt (Tfw) (Bournival et al., 1989). The possibility that CYCLOPS functions in tolerance to $\mathrm{Fol}$ has been ruled out in a separate study (Prihatna et al., 2018). Together, results presented in the current study demonstrate that Solyc08g075770 is the contributor to the Tfw locus identified by Bournival et al. (1989), conferring tolerance to Fol race 3 in 76R which was lost in $r m c$. 


\section{AUTHOR CONTRIBUTIONS}

$\mathrm{CP}$ designed and performed the research, the collection of data, analyzed and interpreted the data, and drafted the manuscript. SB and MB supervised and offered guidance on all aspects of the work and contributed to the writing of the manuscript.

\section{ACKNOWLEDGMENTS}

CP acknowledges the financial assistance of an International SIRF Scholarship funded jointly by the Australian Government and The University of Western Australia. We sincerely thank Daniel

\section{REFERENCES}

Aoki, K., Yano, K., Suzuki, A., Kawamura, S., Sakurai, N., Suda, K., et al. (2010). Large-scale analysis of full-length cDNAs from the tomato (Solanum lycopersicum) cultivar Micro-Tom, a reference system for the Solanaceae genomics. BMC Genomics 11:210. doi: 10.1186/1471-2164-1 $1-210$

Barker, S. J., Edmonds-Tibbett, T. L., Forsyth, L. M., Klingler, J. P., Toussaint, J.P. P., Smith, F. A., et al. (2005). Root infection of the reduced mycorrhizal colonization $(\mathrm{rmc})$ mutant of tomato reveals genetic interaction between symbiosis and parasitism. Physiol. Mol. Plant Pathol. 67, 277-283. doi: 10.1016/ j.pmpp.2006.03.003

Barker, S. J., Stummer, B., Gao, L., Dispain, I., O'connor, P. J., and Smith, S. E. (1998). A mutant in Lycopersicon esculentum Mill. with highly reduced VA mycorrhizal colonization: isolation and preliminary characterisation. Plant J. 15, 791-797. doi: 10.1046/j.1365-313X.1998.00252.x

Biasini, M., Bienert, S., Waterhouse, A., Arnold, K., Studer, G., Schmidt, T., et al. (2014). SWISS-MODEL: modelling protein tertiary and quaternary structure using evolutionary information. Nucleic Acids Res. 42, W252-W258. doi: 10. 1093/nar/gku340

Bohn, G. W., and Tucker, C. M. (1939). Immunity to fusarium wilt in the tomato. Science 89, 603-604. doi: 10.1126/science.89.2322.603

Bournival, B. L., Scott, J. W., and Vallejos, C. E. (1989). An isozyme marker for resistance to race-3 of Fusarium oxysporum f. sp. lycopersici in tomato. Theor. Appl. Genet. 78, 489-494. doi: 10.1007/BF0029 0832

Catanzariti, A.-M., Do, H. T., Bru, P., de Sain, M., Thatcher, L. F., Rep, M., et al. (2017). The tomato I gene for Fusarium wilt resistance encodes an atypical leucine-rich repeat receptor-like protein whose function is nevertheless dependent on SOBIR1 and SERK3/BAK1. Plant J. 89, 1195-1209. doi: 10.1111/ tpj.13458

Catanzariti, A.-M., Lim, G. T., and Jones, D. A. (2015). The tomato I-3 gene: a novel gene for resistance to Fusarium wilt disease. New Phytol. 207, 106-118. doi: $10.1111 /$ nph. 13348

Dietrich, P., Anschütz, U., Kugler, A., and Becker, D. (2010). Physiology and biophysics of plant ligand-gated ion channels. Plant Biol. 12, 80-93. doi: 10. 1111/j.1438-8677.2010.00362.x

Earley, K. W., Haag, J. R., Pontes, O., Opper, K., Juehne, T., Song, K. M., et al. (2006). Gateway-compatible vectors for plant functional genomics and proteomics. Plant J. 45, 616-629. doi: 10.1111/j.1365-313X.2005. 02617.x

Fuchs, J. G., Moënne-Loccoz, Y., and Défago, G. (1997). Nonpathogenic Fusarium oxysporum strain Fo47 induces resistance to Fusarium wilt in tomato. Plant Dis. 81, 492-496. doi: 10.1094/PDIS.1997.81.5.492

Gao, L.-L., Delp, G., and Smith, S.E. (2001). Colonization patterns in a mycorrhizadefective mutant tomato vary with different arbuscular-mycorrhizal fungi. New Phytol. 151, 477-491. doi: 10.1046/j.0028-646x.2001.00193.x

Gonzalez-Cendales, Y., Catanzariti, A.-M., Baker, B., Mcgrath, D. J., and Jones, D. A. (2016). Identification of I-7 expands the repertoire of genes for resistance to Fusarium wilt in tomato to three resistance gene classes. Mol. Plant Pathol. 17, 448-463. doi: $10.1111 / \mathrm{mpp} .12294$
Ruzicka (Donald Danforth Plant Science Center, St. Louis, MO, United States) for the pEarleyGate-Solyc08g075770 vector and his encouragement to test its function. We also thank Simon Schiml and Holger Puchta (Botanical Institute II, Karlsruhe Institute of Technology, Germany) for pDe-CAS9 and pEn-C1.1 vectors.

\section{SUPPLEMENTARY MATERIAL}

The Supplementary Material for this article can be found online at: https://www.frontiersin.org/articles/10.3389/fmicb. 2018.01226/full\#supplementary-material

Guex, N., Peitsch, M. C., and Schwede, T. (2009). Automated comparative protein structure modeling with SWISS-MODEL and Swiss-PdbViewer: a historical perspective. Electrophoresis 30, S162-S173. doi: 10.1002/elps.200900140

Houterman, P. M., Cornelissen, B. J. C., and Rep, M. (2008). Suppression of plant resistance gene-based immunity by a fungal effector. PLoS Pathog. 4:e1000061. doi: 10.1371/journal.ppat.1000061

Jones, D. T., Taylor, W. R., and Thornton, J. M. (1992). The rapid generation of mutation data matrices from protein sequences. Bioinformatics 8, 275-282. doi: 10.1093/bioinformatics/8.3.275

Kumar, S., Stecher, G., and Tamura, K. (2016). MEGA7: molecular evolutionary genetics analysis version 7.0 for bigger datasets. Mol. Biol. Evol. 33, 1870-1874. doi: 10.1093/molbev/msw054

Larkan, N. J., Ruzicka, D. R., Edmonds-Tibbett, T., Durkin, J. M., Jackson, L. E., and Smith, F. A. (2013). The reduced mycorrhizal colonisation ( $\mathrm{rmc}$ ) mutation of tomato disrupts five gene sequences including the CYCLOPS/IPD3 homologue. Mycorrhiza 23, 573-584. doi: 10.1007/s00572-0130498-7

Larkan, N. J., Smith, S. E., and Barker, S. J. (2007). Position of the reduced mycorrhizal colonisation (Rmc) locus on the tomato genome map. Mycorrhiza 17, 311-318. doi: 10.1007/s00572-007-0106-9

Lazo, G. R., Stein, P. A., and Ludwig, R. A. (1991). A DNA transformationcompetent Arabidopsis genomic library in Agrobacterium. Biotechnology 9, 963-967. doi: 10.1038/nbt1091-963

Liu, P.-L., Du, L., Huang, Y., Gao, S.-M., and Yu, M. (2017). Origin and diversification of leucine-rich repeat receptor-like protein kinase (LRR-RLK) genes in plants. BMC Evol. Biol. 17:47. doi: 10.1186/s12862-017-0891-5

Mayer, K., Schuller, C., Wambutt, R., Murphy, G., Volckaert, G., Pohl, T., et al. (1999). Sequence and analysis of chromosome 4 of the plant Arabidopsis thaliana. Nature 402, 769-777. doi: 10.1038/47134

Moeder, W., Urquhart, W., Ung, H., and Yoshioka, K. (2011). The role of cyclic nucleotide-gated ion channels in plant immunity. Mol. Plant 4, 442-452. doi: $10.1093 / \mathrm{mp} / \mathrm{ssr} 018$

Murray, M. G., and Thompson, W. F. (1980). Rapid isolation of high molecular weight plant DNA. Nucleic Acids Res. 8, 4321-4325. doi: 10.1093/nar/8.19.4321

Olivain, C., and Alabouvette, C. (1999). Process of tomato root colonization by a pathogenic strain of Fusarium oxysporum f. sp. lycopersici in comparison with a non-pathogenic strain. New Phytol. 141, 497-510. doi: 10.1046/j.1469-8137. 1999.00365.x

Prihatna, C., Larkan, N. J., Barbetti, M. J., and Barker, S.J. (2018). Tomato CYCLOPS/IPD3 is required for mycorrhizal symbiosis but not tolerance to Fusarium wilt in mycorrhizal-deficient tomato mutant rmc. Mycorrhiza (in press).

R Core Team (2016). R: A Language and Environment for Statistical Computing. Vienna: R Foundation for Statistical Computing.

Schiml, S., Fauser, F., and Puchta, H. (2014). The CRISPR/Cas system can be used as nuclease for in planta gene targeting and as paired nickases for directed mutagenesis in Arabidopsis resulting in heritable progeny. Plant J. 80, 1139-1150. doi: 10.1111/tpj.12704

Schindelin, J., Arganda-Carreras, I., Frise, E., Kaynig, V., Longair, M., Pietzsch, T., et al. (2012). Fiji: an open-source platform for biological-image analysis. Nat. Methods 9, 676-682. doi: 10.1038/nmeth.2019 
Sievers, F., Wilm, A., Dineen, D., Gibson, T. J., Karplus, K., Li, W., et al. (2011). Fast, scalable generation of high-quality protein multiple sequence alignments using Clustal Omega. Mol. Syst. Biol. 7:539. doi: 10.1038/msb.2011.75

Simons, G., Groenendijk, J., Wijbrandi, J., Reijans, M., Groenen, J., Diergaarde, P., et al. (1998). Dissection of the Fusarium I2 Gene Cluster in tomato reveals six homologs and one active gene copy. Plant Cell 10, 1055-1068. doi: 10.2307/ 3870690

Stall, R., and Walter, J. (1965). Selection and inheritance of resistance in tomato to isolates of races 1 and 2 of the Fusarium wilt organism. Phytopathology 55, 1213-1215.

Takken, F., and Rep, M. (2010). The arms race between tomato and Fusarium oxysporum. Mol. Plant Pathol. 11, 309-314. doi: 10.1111/j.1364-3703.2009. 00605. $\mathrm{x}$

Vierheilig, H., Coughlan, A. P., Wyss, U., and Piché, Y. (1998). Ink and vinegar, a simple staining technique for arbuscular-mycorrhizal fungi. Appl. Environ. Microbiol. 64, 5004-5007.

Waterhouse, A. M., Procter, J. B., Martin, D. M., Clamp, M., and Barton, G. J. (2009). Jalview Version 2-a multiple sequence alignment editor and analysis workbench. Bioinformatics 25, 1189-1191. doi: 10.1093/bioinformatics/ btp033

Wei, Z., Wang, J., Yang, S., and Song, Y. (2015). Identification and expression analysis of the LRR-RLK gene family in tomato (Solanum lycopersicum) Heinz 1706. Genome 58, 121-134. doi: 10.1139/gen-20150035

Conflict of Interest Statement: The authors declare that the research was conducted in the absence of any commercial or financial relationships that could be construed as a potential conflict of interest.

Copyright (c) 2018 Prihatna, Barbetti and Barker. This is an open-access article distributed under the terms of the Creative Commons Attribution License (CC BY).

The use, distribution or reproduction in other forums is permitted, provided the original author(s) and the copyright owner are credited and that the original publication in this journal is cited, in accordance with accepted academic practice. No use, distribution or reproduction is permitted which does not comply with these terms. 